\title{
Subliminal perception can be predicted from prestimulus activity
}

\author{
Henry Railo $1,2,3,{ }^{*}$, Roberto Piccin ${ }^{4}$, \& Karolina M. Lukasik ${ }^{5}$
}

${ }^{1}$ Department of Clinical Neurophysiology, University of Turku, 20521, Turku, Finland

${ }^{2}$ Turku Brain and Mind Centre, University of Turku, Turku, Finland

${ }^{3}$ Department of Psychology, University of Turku, Turku, 20014, Finland

${ }^{4}$ Department of Life Sciences, University of Trieste, 34127 Trieste, Italy

${ }^{5}$ Department of Psychology, Åbo Akademi University, 20500 Turku, Finland

* Corresponding author:

Henry Railo,

Department of Clinical Neurophysiology,

University of Turku and Turku University Hospital,

Finland,

henry.railo@utu.fi

Running title: Subliminal vision

Acknowledgments: The study was funded by the Academy of Finland (grant \#308533). The authors also thank two reviewers for very helpful comments.

\section{Conflict of Interest Statement: none}

\section{Abstract}

Humans sometimes make accurate guesses about stimuli they report not consciously seeing-this phenomenon is known as "subliminal perception." We asked participants $(\mathrm{N}=31)$ to discriminate the location of a briefly presented low-contrast visual stimulus, and then rate how well they saw the stimulus. The behavioral accuracy of discriminating the location of a subjectively subliminal stimulus could be predicted in a trial-by-trial manner from lateralized low frequency $(1-15 \mathrm{~Hz})$ electroencephalographic (EEG) activity before the stimulus. This effect was observed up to $1 \mathrm{~s}$ before the stimulus was presented. Lateralized occipital prestimulus EEG power modulated the stimulus-evoked activity in a complex manner, but the amplitude of the stimulus-evoked electrophysiological response was not strongly modulated by subliminal objective discrimination accuracy. Signal detection analyses indicated that the participants' capacity to discriminate subliminal stimuli lay on the same continuum as conscious vision. The results suggest that subliminal perception is not an automatic stimulus-evoked process but relies on perceptual decisions about weak perceptual signals that may be available for introspection.

Key words: Subliminal perception, unconscious perception, consciousness, blindsight, prestimulus, oscillations, EEG, cortical excitability 


\section{Introduction}

One of the major aims of neuroscience is to uncover which neural processes enable a person to consciously experience and respond to stimuli in their environment. Interestingly, visual stimuli that individuals report as not consciously perceived can influence their behavior, a phenomenon known as "subliminal perception" (Hannula, Simons, \& Cohen, 2005; Kouider \& Dehaene, 2007). The neural mechanisms that enable this subliminal capacity are not well understood. Traditional models of subliminal perception emphasize stimulus-evoked activity, but here, we draw attention to the possibility that fluctuations in spontaneous neural activity before stimulus onset may be important for subliminal vision. We use the term "subliminal perception" instead of "unconscious perception" to emphasize that stimuli the participants report not seeing may only be below the criterion they use to categorize a stimulus as "seen". Alternatively, subliminal perception could be a perceptual capacity that is clearly separate from conscious perception. If this latter case is true, then performance on subliminal trials should be independent of introspective reports, and based on altogether different neural mechanisms than conscious vision.

According to the currently popular models of vision, the feedforward sweep of stimulustriggered activation through cortical areas happens outside of the individual's consciousness, but this activation is assumed to have the power to influence behavior (Vanrullen, 2007; VanRullen \& Koch, 2003). The extent to which feedforward processes enable subliminal contents to influence behavior may depend on selective attention: attended stimuli may produce stronger activation, increasing the likelihood of correct behavioral response (Dehaene, Changeux, Naccache, Sackur, \& Sergent, 2006). Feedforward activation may also trigger the formation of recurrent activity loops between different cortical areas, enabling stronger subliminal perceptual capacity (Dehaene et al., 2006). These conclusions are supported by studies that show that under certain conditions where the participants report not seeing a stimulus, visually evoked activation is preserved but does not ignite widespread cortical activation (Gaillard et al., 2009; Kouider, Dehaene, Jobert, \& Le Bihan, 2007; Salti et al., 2015; Supèr, Spekreijse, \& Lamme, 2001). Widespread cortical activation is assumed to enable full-fledged conscious perception, allowing the participant to guide behavior based on the conscious contents and, for example, report what they perceived (i.e. "access consciousness"; Dehaene \& Changeux, 2011; Lamme \& Roelfsema, 2000).

If the accuracy of subliminal perceptual responses depends on the strength of the early visual activation (Dehaene et al., 2006), then visually evoked activation should be higher in the trials where the participants provide a correct answer. Some studies that show enhanced eventrelated potentials (ERP) around $200 \mathrm{~ms}$ after stimulus onset in occipitotemporal electrodes when the participants correctly discriminate a target despite claiming that they do not consciously see it support this conclusion (Koivisto \& Grassini, 2016; Lamy, Salti, \& Bar-Haim, 2009). However, because reports of conscious vision correlate with ERP amplitude in the same time window-an effect known as visual awareness negativity (Förster, Koivisto, \& Revonsuo, 2020; Railo, Koivisto, \& Revonsuo, 2011) - this correlate of subliminal perceptions could reflect degraded conscious perception rather than strictly unconscious perception. Koivisto and Grassini (2016) reported that the ERP correlate of subliminal discrimination also correlated with conservative response criterion, suggesting that what the participants labeled "not seen" may have been severely degraded conscious perception. This is consistent with the results of Peters and Lau (2015), which suggest that when perception is measured using a procedure that minimizes the influence of criterion setting, no evidence for strictly subliminal perception is observed.

Preserved or enhanced early visually evoked neural activation in subliminal trials may not alone explain why in some subliminal trials the participant responds correctly but not in other trials. In contrast to the idea that the strength of early visually evoked responses correlates with the likelihood of correct behavioral responses within reportedly subliminal trials, Salti et al. (2012) found that only the late P3 wave correlated with the accuracy of subliminal discrimination (see also, (Salti et al., 2015)). This could mean that subliminal perception may be less influenced by the 
strength of the visually evoked response than currently assumed. Possibly, the trial-by-trial variation in behavioral performance on subliminal trials relates not to the strength of visual-evoked response but to how well decision-making processes can "access" the visual stimulus-related information.

Instead of focusing on visually evoked activity, we reasoned that subliminal perception could be explained by spontaneous variation in neural activity before stimulus presentation. Indeed, recent studies have emphasized the importance of top-down modulation of visual cortical activity on visual processing (Muckli \& Petro, 2013). Prestimulus oscillations may bias the processing of a stimulus by modulating how excitable cortical neurons are, for instance, to incoming visual stimulus (Samaha, Iemi, Haegens, \& Busch, 2020). Prestimulus oscillations could influence whether subliminal stimuli elicit the correct behavioral response for at least two reasons. First, if subliminal perception can be explained in terms of the strength of the early evoked response, this effect could be driven by the prestimulus brain state: in trials where the participant's attentional state before stimulus presentation is optimal, the stimulus may trigger stronger visually evoked responses, thereby enabling more successful behavioral performance, even if the participants reported not seeing the stimulus. Numerous studies have shown that conscious visual perception is strongly influenced by low-frequency oscillations, especially the alpha $(8-13 \mathrm{~Hz})$ oscillations, before stimulus presentation (Bareither, Chaumon, Bernasconi, Villringer, \& Busch, 2014; Boncompte, Villena-González, Cosmelli, \& López, 2016; Britz, Hernàndez, Ro, \& Michel, 2014; Iemi \& Busch, 2018; Jensen, Bonnefond, \& VanRullen, 2012; Kloosterman et al., 2019; Limbach \& Corballis, 2016; Mathewson, Gratton, Fabiani, Beck, \& Ro, 2009; Samaha, Iemi, \& Postle, 2017; Schroeder \& Lakatos, 2009; Thut, Nietzel, Brandt, \& Pascual-Leone, 2006). However, prestimulus alpha power is currently assumed to influence subjective detection of stimuli, and not perceptual discrimination (Benwell et al., 2017; Iemi, Chaumon, Crouzet, \& Busch, 2017; Samaha et al., 2020).

Second, prestimulus neural activity could explain variations in perceptual decision making instead of directly influencing the visually evoked neural response. Baria, Maniscalco, and $\mathrm{He}$ (2017) showed that when the prestimulus activity patterns resemble a pattern evoked by the visual stimulus, correct behavioral discrimination is more likely, even when the participants report not consciously perceiving the stimulus. This type of explanation builds on the idea that when the participants report not seeing a stimulus - that is, when they subjectively have no stimulus-related information on what to base their response on - the response is determined largely by the prestimulus state. This reasoning is very different from current models of (un)conscious vision (Dehaene et al., 2006; Lamme \& Roelfsema, 2000), which attribute the differences in responding to stimulus-evoked response strength.

In the current study, we randomly presented participants with a threshold-level low-contrast stimulus on either the left or right visual field and asked them to report in which visual hemifield the stimulus was presented and then rate how well they saw it (Figure 1A). We reasoned that we would have the highest chance of observing subliminal perception-possibly an unconscious capacity that is independent of conscious perception - using this type of simple location discrimination task. We first used signal detection theoretic measures to test if subliminal perception is independent of subjectively reported visibility. We then used single-trial analyses to test whether prestimulus EEG oscillatory power predicts trial-by-trial variation in behavioral performance, and stimulus-evoked activation. The left versus right location discrimination task allowed us to take into account if the lateralized EEG activity predicts performance in subliminal trials.

\section{Materials and Methods}

Participants 
Thirty-four students (3/34 males, 3/34 left-handed) with a mean age of $24.4(\mathrm{SD}=3.6)$ years and no neurological disorders participated in the experiment. Three participants were excluded before the EEG preprocessing because of a high false alarm rate (participants with false alarm rate $>0.3$ were excluded; mean false alarm rate of the excluded participants was $0.59, \mathrm{SD}=0.26$ ). The participants were students at the University of Turku. Each participant gave written informed consent. The study was conducted in accordance with the Declaration of Helsinki and was approved by the ethics committee of the Hospital District of Southwest Finland.

\section{Stimuli}

The experiment was run in MATLAB (version R2014b) using the Psychophysics Toolbox (Brainard, 1997). Stimuli were presented on an VIEWPixx/EEG LCD monitor with a $120 \mathrm{~Hz}$ refresh rate. The stimuli were Gabor patches (a diameter of $6.5^{\circ}$ of visual angle and a frequency of 0.7 cycles/degree; the phase and orientation of the Gabor patch was randomly varied on each trial) presented on a gray $50 \%$ background $\left(45.5 \mathrm{~cd} / \mathrm{m}^{2}\right)$. On two-thirds of the trials, a low-contrast Gabor patch was presented. The intensity of this low-contrast Gabor was individually adjusted to be near a 50\% subjective detection threshold using a QUEST staircase (Watson \& Pelli, 1983). On one-sixth of the trials, a high-contrast Gabor with three times higher contrast than the contrast of the lowcontrast Gabor was presented. The high-contrast stimuli were presented to allow the participant to clearly see the stimuli every now and then, meaning they could use the whole visibility rating scale. Both low-contrast and high-contrast stimuli were presented for a duration of two frames (16.6 ms in total). One-sixth of the trials did not have any stimulus (catch trials), instead containing a blank screen with the fixation point lasting two frames. Only trials with the low-contrast stimuli were included in the statistical analysis.

\section{Experimental design}

The stimulus was presented after a fixation period that varied from $668 \mathrm{~ms}$ to $1332 \mathrm{~ms}$ from trial to trial to prevent the participant from learning to expect the stimulus at a specific delay. $250 \mathrm{~ms}$ after the Gabor was presented (or catch trial), the fixation point turned into an arrow pointing left and right, indicating that the participant should try to report the side of the target. After this, the numbers " $0-1-2-3$ " were presented on the center of the screen to prompt a visibility rating response. Both responses were given by pressing a key on a numpad. The participants were told to try to give their best guess about stimulus location, even when they felt they did not see any stimulus. The visibility rating (Overgaard, 2011; Ramsøy \& Overgaard, 2004; Sandberg, Timmermans, Overgaard, \& Cleeremans, 2010) was given using a four-step scale where the alternatives were as follows: 0) "did not see stimulus at all," 1) "not sure but possibly saw something," 2) "pretty sure I saw it," and 3) "saw the stimulus clearly." The difference between alternatives 0 and 1 was stressed to the participant; that is, we emphasized that if they felt that they saw any glimpse of the stimulus, even an extremely weak one, they should select the second lowest alternative. The rating task was preferred over a yes/no task to make sure that the participants used a strict criterion for reporting stimuli "subliminally". Throughout the present study, a participant is assumed to be subliminal of a stimulus only when she/he chose the lowest visibility rating (the three higher alternatives are all counted as conscious). No instruction was given about the response speed (of either response). The participants were also told that in some trials, the stimulus would not be presented, and they were told that on these trials, the correct response was to report that they did not see the stimulus. The experiment comprised a total of 400 trials per participant, subdivided into 10 blocks of 40 trials each.

\section{EEG recording and preprocessing}

A 64-channel EEG was recorded at a $500 \mathrm{~Hz}$ sampling rate with a NeurOne Tesla amplifier. Before the recording, electrode impedances were brought near $5 \mathrm{k} \Omega$. Two additional electrodes were used 
to record electro-oculograms (EOG): one electrode was placed beneath and the other beside the participant's left eye.

Preprocessing was carried out with MATLAB (version R2016b) using the EEGlab toolbox (version 14.1.1b). For each participant, bad channels (channels with no response or very noisy signals) were manually checked and interpolated. EEG data were re-referenced to the average of all electrodes. A $0.25 \mathrm{~Hz}$ high-pass filter and an $80 \mathrm{~Hz}$ low-pass filter were applied to the data. Line noise was cleaned up using the CleanLine EEGlab plugin. Data were epoched from $-2000 \mathrm{~ms}$ to $1500 \mathrm{~ms}$ relative to the stimulus onset. Trials with eye movements (EOG electrodes) within $-500 \mathrm{~ms}$ to $500 \mathrm{~ms}$ relative to the stimulus onset were discarded (on average, 24 trials, $\mathrm{SD}=37$ trials were rejected per participant). The locations of the electrodes were transposed so that the left hemisphere was always contralateral and the right hemisphere always ipsilateral relative to stimulus presentation. A time frequency analysis was performed on single trials using complex Morlet wavelets (frequency range: $1-30 \mathrm{~Hz}$ with $2 \mathrm{~Hz}$ step size, with the number of cycles increasing linearly from 1 to 12). No baseline correction was applied because we examined prestimulus oscillations. Note that due to the limited temporal resolution of the wavelet analysis, variation in poststimulus time windows could influence the results of prestimulus time points, especially for low $(<3 \mathrm{~Hz})$ frequencies. Our study was designed to examine alpha oscillations in particular. With the present parameters of wavelet analysis, the contamination of prestimulus alpha time-points by poststimulus effects should be minimal for prestimulus time-points before $-150 \mathrm{~ms}$ (see, Iemi et al., 2017, for details).

\section{Statistical analysis}

Behavioral signal detection measures of sensitivity (d') and criterion (c) were calculated from binarized subjective visibility ratings. A hit was defined as a visibility rating of $1-3$ when a stimulus was presented. False alarms were trials where the participant reported seeing a target (rating 1-3) when no stimulus was presented.

Time points between $1000-0 \mathrm{~ms}$ before stimulus onset in steps of $24 \mathrm{~ms}$ were statistically analyzed to examine if prestimulus oscillatory power predicted behavioral performance. These analyses were performed in a mass univariate manner (Maris \& Oostenveld, 2007), analyzing all the time points and all frequencies between 1 and $30 \mathrm{~Hz}$. Statistical analyses were performed on the single-trial data. We used mixed-effects logit models to test whether log transformed oscillatory power at specific time point and frequency predicted the accuracy of the response (correct/incorrect) or subjective visibility (saw/did not see). In the mass univariate models, the participants were defined as random factors, and the intercepts were allowed to vary between participants. More complicated models often failed to converge, and the models also took very long to compute. Log transformed power was used as the predictor because untransformed data were strongly right skewed.

Statistical significance was assessed using permutation testing by randomly shuffling condition labels (1000 iterations). We used threshold free cluster enhancement (TFCE) to take into account the clustering of effects in neighboring time points and frequency bands (Smith \& Nichols, 2009). Maps of the $t$ values obtained from the mass univariate analyses were TFCE transformed using a function from the Limo EEG package (Pernet, Chauveau, Gaspar, \& Rousselet, 2011). TFCE was performed for the authentic data and also after each permutation. The highest TFCE score $\left(\mathrm{TFCE}_{\max }\right.$ ) of each permutation was saved to obtain a null distribution corrected for multiple comparisons. This analysis was run separately on data from five clusters of electrodes. To ensure two-tailed testing, and take into account that analysis was repeated five times (for each cluster of channels), we further used a Bonferroni corrected alpha level to assess statistical significance. TFCE values with the probability $<.005$ when compared with the $\mathrm{TFCE}_{\max }$ distribution were considered statistically significant. 
We also analyzed ERPs and event-related spectral perturbations (ERSP) to examine how behavioral performance (subjective and objective measures) was associated with stimulus-evoked responses. These analyses were performed using a similar mass univariate approach as described earlier (mixed-effects logit models with random intercepts, 1000 permutations, and TFCE, and $\mathrm{p}=$ .005 alpha level). In the ERP analyses TFCE was calculated over neighboring time samples and electrodes. Before ERP analysis, EEG was low-pass filtered at $40 \mathrm{~Hz}$. Single-trial ERPs were baseline corrected to $-200 \mathrm{~ms}$. In standard ERSP analysis, multiple trials are averaged, and it is measured how EEG oscillatory power changes in response to a stimulus relative to some baseline state. Because we performed the ERSP analysis on single trials, we centered the oscillatory power at each poststimulus time point and frequency relative to the average power of a frequency band during the whole trial ( -2 to 1.5 s relative to stimulus onset) (Grandchamp \& Delorme, 2011).

Number of trials per participant in the statistical analyses are as follows. Participants had on average 125 trials in the conscious condition $(\mathrm{SD}=42$ trials), and $120(\mathrm{SD}=39)$ trials in the subliminal condition. When both seen and unseen trial are included in the analysis, the comparison of correct vs. incorrect objective discrimination is based on $190(\mathrm{SD}=37) \mathrm{vs} .55(\mathrm{SD}=24)$ trials per participant on average, respectively. The main analysis of objective discrimination performance on subliminal trials is based on average on 71 (correct response, $\mathrm{SD}=23$ ) vs. 49 (incorrect response, $\mathrm{SD}=20$ ) trials per participant.

Datasets are available for download at https://osf.io/xz8jr/.

\section{Results}

\section{Behavioral results}

On average, the participants reported seeing the target (visibility rating 1-3) in 51\% of the trials $(\mathrm{SEM}=14 \%)$ when the stimulus was presented. When the stimulus was not presented, they rarely reported seeing the target $(9 \%, \mathrm{SEM}=8 \%)$. When the participants reported not seeing the target at all (visibility rating $=0$ ), they nevertheless correctly reported the side of the stimulus in $60 \%$ of the trials on average ( $\mathrm{t}$ test against $50 \%$ chance-level: $\mathrm{t}=5.95, \mathrm{df}=30, \mathrm{p}=1.60 \times 10^{-6}$ )-we refer to this as "subliminal perception".
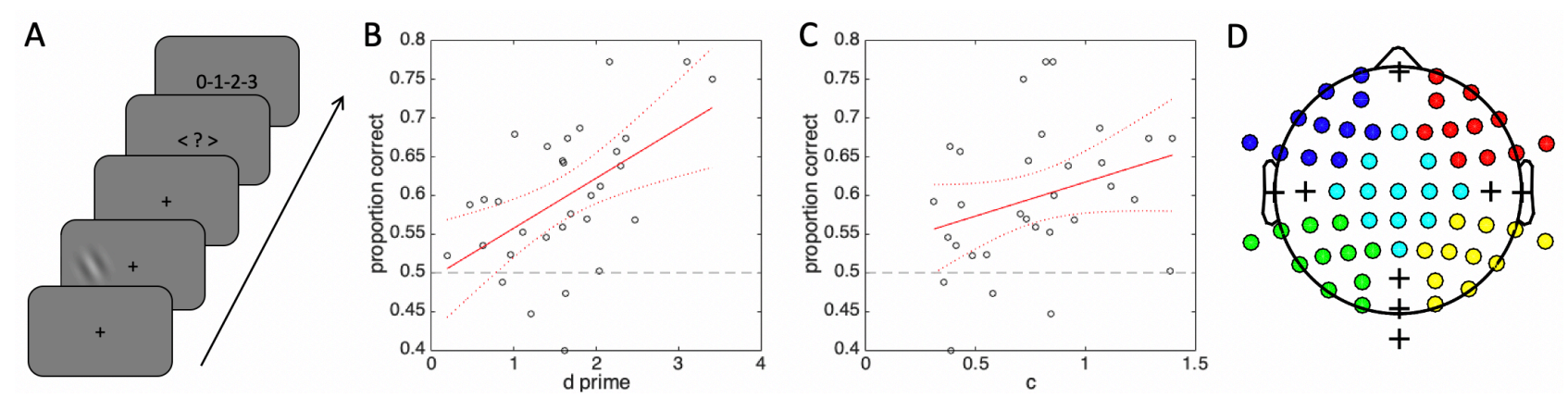

Figure 1. Behavioral paradigm and results. A) A schematic of a single trial: the participants gave a forced discrimination response and rated how well they saw the stimulus. B) Correlation between the sensitivity of subjectively detecting the stimulus and proportion correct (forced discrimination task) on subliminal trials. C) Correlation between criterion for reporting conscious perception and the correct proportion on subliminal trials. D) Electrode clusters used in EEG analysis. Each color represents one cluster. Plus-symbols are electrodes that are not part of any cluster.

Does accurate responses in the trials where the participants report no conscious perception reveal the existence of a perceptual capacity that functions independently of conscious vision? We calculated the participants' sensitivity and criterion for consciously detecting the target (i.e., the measures were calculated from subjective reports, not objective performance, using signal detection analyses). As shown in Figure 1B, the participants who were not sensitive to subjectively detecting the stimulus $\left(\mathrm{d}^{\prime} \approx 0\right)$ did not reveal subliminal perception (intercept of the linear model $=.49$ ). As 
shown in Figure 1C, the participants also tended to use a conservative criterion for reporting conscious perception. This is common with low-contrast stimuli because the participants try to minimize false alarms. Based on extrapolating the fitted linear model, if the participants used statistically optimal criterion $(\mathrm{c}=0)$ for reporting consciousness, they would also have performed at near the chance level in discriminating the stimulus side of the subliminal trials (intercept $=.53$ ). These results indicate that objective discrimination was not separate from subjectively reported vision.

\section{Prestimulus correlates}

We tested if prestimulus EEG power predicted the participants' objective discrimination performance when they subjectively reported not seeing the target at all (the lowest visibility rating). Because the lateralization of cortical excitability varies depending on whether attention is focused toward the right or left visual hemifield (Boncompte et al., 2016; Thut et al., 2006; Worden, Foxe, Wang, \& Simpson, 2000), we took this into account by transposing the electrode locations for each trial so that the left electrode locations represent electrodes that were contralateral with respect to the visual stimulus (and right electrodes were ipsilateral). The electrode clusters are visualized in Fig. 1D.
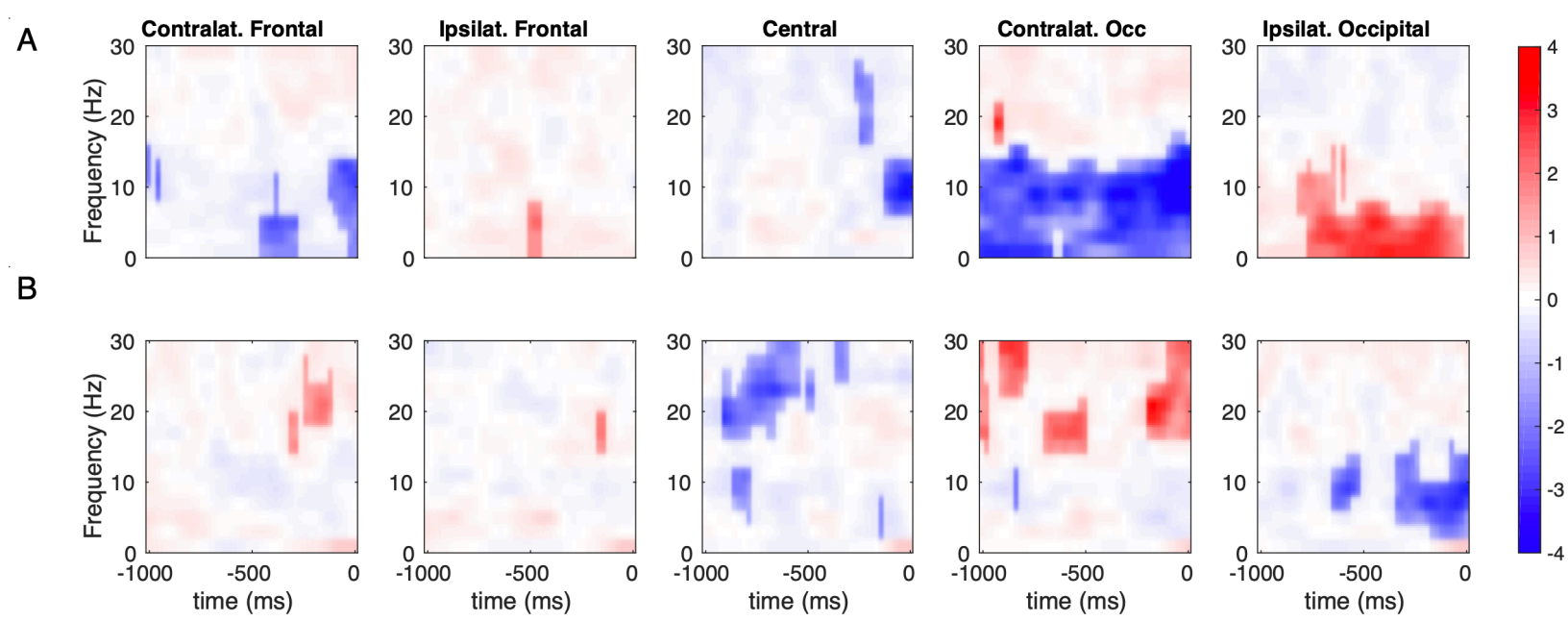

Figure 2. How prestimulus oscillatory power predicts behavior. Results of mass univariate mixedeffects logit models where a single trial's prestimulus EEG power was used to predict $A$ ) the location discrimination accuracy on subliminal trials (visibility $=0$ ) and B) binarized visibility ratings (did not see $=0 ; 1-3=$ saw). Color is the $t$ value of the mixed-effects logit model. The blue color denotes a negative correlation and red a positive correlation. The faint colors are time points/frequencies where the model does not statistically significantly predict behavior. Bright colors show statistically significant modulation according to TFCE and permutation testing. Different columns represent different clusters of electrodes.

As shown in Figure 2A, objective performance on trials where the participants reported not seeing the stimulus at all could be predicted from prestimulus power. Oscillatory power at the occipital electrode clusters predicted the accuracy of responses up to $1 \mathrm{~s}$ before the stimulus was presented, even though the participants reported just guessing. The effect is clearly lateralized: lower oscillatory power $(1-15 \mathrm{~Hz})$ contralateral to the stimulus and higher oscillatory power (1-5 $\mathrm{Hz}$ ) in ipsilateral occipital electrodes predicts better performance. The strongest effects are observed in the occipital electrode clusters, but the frontal and central electrode clusters also show some effects. The results are relatively similar when consciously seen trials are included in the analysis (Supplementary Figure 1). When electrode locations were not transposed, only a prestimulus effect 
in the alpha band in occipital electrodes (up to $250 \mathrm{~ms}$ before stimulus onset) was observed (Supplementary Figure 2).

The prestimulus power in single trials was also associated with reported conscious visibility of the target in a trial-by-trial manner, as shown in Figure 2B. For instance, in the ipsilateral occipital electrode clusters, lower power of around 5-10 Hz right before stimulus onset predicted conscious perception. Unlike in the case of accuracy of responding in subliminal trials, this effect does not show lateralization. A similar effect is also found when only trials with the correct objective discrimination accuracy are included in the analysis (Supplementary Figure 4). When non-transposed electrode locations were used, the prestimulus power around $10 \mathrm{~Hz}$ predicted reported visibility (Supplementary Figure 3).

The above results show that behavioral performance correlates with prestimulus power in subliminal trials. To see how well the participants' subliminal visual discrimination responses in single trials could be predicted based on the prestimulus oscillatory power, we ran a mixed-effects logit model that included the average power of the ipsilateral electrode cluster 668-190 ms before stimulus onset at $3 \mathrm{~Hz}$ and the average power in the contralateral electrode cluster 190-71 ms before stimulus onset across 5-9 Hz (but not their interaction). These frequencies/time windows were selected because the strongest prestimulus effects cluster around them. The random subject structure of the logit model included separate intercepts and slopes for both ipsilateral and contralateral power for each participant. This classifier's area-under-the-curve (AUC) was $65 \%$, which is very good performance given that the model was classifying the responses that the participants subjectively labeled as guesses. Interestingly, the interaction between the contralateral and ipsilateral cluster predictors did not increase classifier performance (interaction $t=-0.44$ ). This suggests that the prestimulus power in the contralateral and ipsilateral sites contributed independently to discrimination accuracy. The AUC of the classifier was clearly statistically significant when compared to a null distribution obtained by 1000 permutations with randomly shuffled observations. The highest AUC obtained with the randomly shuffled data was 59\% (mean AUC with permuted data was 54\%).

\section{Event-related potentials}

What is the mechanism through which spontaneous prestimulus power influences the discrimination of subliminal stimuli? High-amplitude low-frequency oscillations have been associated with suppressing the processing of nonrelevant signals, whereas low-amplitude low-frequency power is associated with amplifying sensory input (Schroeder \& Lakatos, 2009). The lateralized contribution of an occipital prestimulus oscillatory power on subliminal discrimination performance suggests that successful behavioral responding in subliminal trials may be related to enabling strong responses to the (contralateral) visual stimulus while suppressing unwanted signals in the opposite (ipsilateral) visual field (where no target is presented). If this is the case, we should observe enhanced visually evoked responses to correctly discriminated subliminal targets (when compared with the trials of incorrect responses).

To examine how objective discrimination accuracy and subjective visibility of the stimulus influences stimulus-evoked activity, we analyzed ERPs in a single-trial manner. As in the prestimulus analyses, the electrode locations in these analyses were transposed so that in the scalp maps, the left hemisphere locations would correspond to the contralateral hemifield. As shown in Figure 3A, subjective visibility of the stimulus was associated with a strong increase of ERP amplitudes $275 \mathrm{~ms}$ after stimulus onset, spanning most electrode locations. Nearly identical effects are produced by when objective discrimination performance is examined (all trials included, Supplementary Figure 5), in line with the behavioral result that objective discrimination was not independent of subjectively reported vision. However, as shown in Fig. 3B, no strong effects are seen in ERPs when the analysis is restricted to subliminal trials. The only effect approaching statistical significance is enhanced late ERPs (around $475 \mathrm{~ms}$ ) to targets that were correctly 
discriminated, despite being reported subliminal. Therefore, these results do not support the conclusion that successful subliminal perceptual discrimination is enabled by a stronger visually evoked neural response.
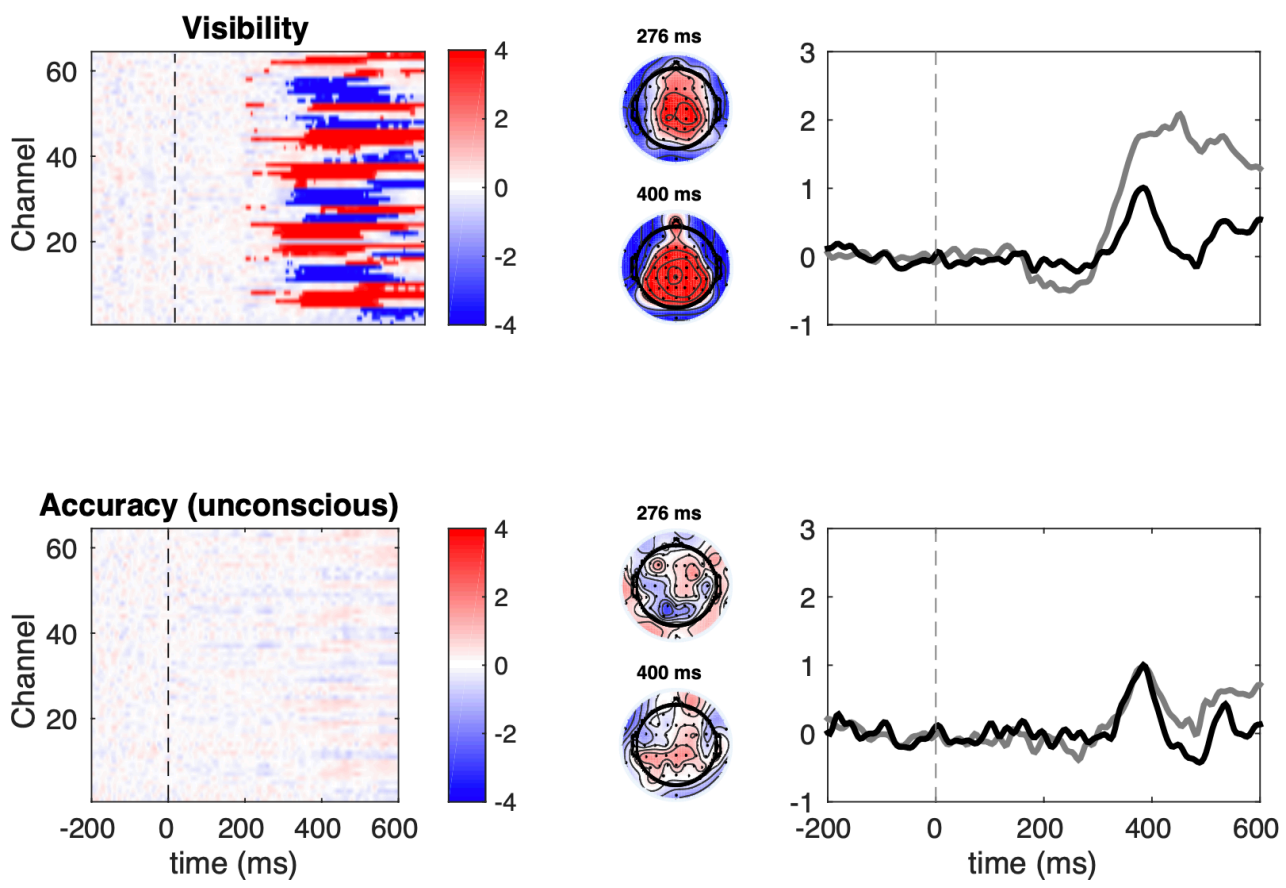

Figure 3. Event-related potentials. A) Single-trial visibility ratings (top panels) predicted ERPs 275 ms after stimulus onset. B) When only reportedly subliminal trials were included in the analysis (bottom panels), no strong effects were observed. The figures on the left show the results of the mass univariate mixed-effects regression models. Color denotes $t$ value, and statistically significant effects (based on TFCE) are shown with a bright color. The middle panels show scalp topographies of the effects at $276 \mathrm{~ms}$ and $400 \mathrm{~ms}$ after stimulus onset (note that the electrode locations have been transposed: electrodes over the left scalp locations are contralateral to the stimulus). The rightmost panels show ERPs measured at the contralateral occipital cluster. The gray line depicts consciously seen (top panels) or correctly discriminated (bottom panels) trials, and the black line depicts not consciously seen (top panels)/incorrectly discriminated trials (bottom panels).

Next, we asked how prestimulus oscillatory power influences ERPs. If the prestimulus process that mediates accurate subliminal discrimination relates to fluctuations in cortical excitability, we should be able to observe that the contralateral occipital power is inversely related to ERP amplitude. We examined whether single-trial oscillatory power in the same frequencies/time windows that predicted objective behavioral performance (ipsilateral electrode cluster: 668-190 ms before stimulus onset at $3 \mathrm{~Hz}$ and contralateral electrode cluster: 190-71 ms before stimulus onset at 5-9 Hz) also predicted ERP amplitude. We first included all trials (subliminal and conscious) in the analysis. These results are shown in Figure 4A. Contralateral occipital prestimulus power predicted ERP amplitude at time points between 0-400 ms after stimulus onset. Starting at an early time-window (around $125 \mathrm{~ms}$ ), contralateral occipital prestimulus power positively correlated with ERPs in contralateral occipito-temporal electrodes (red color in Figure 4A). Contralateral occipital prestimulus power correlated negatively with ERPs in frontal electrodes (i.e., blue color in Figure 4A). This effect is consistent with the observed relationship between ERPs and visual behavioral performance: As shown in Figure 3, better behavioral performance is associated with more positive ERPs in frontal sites between 275-400 ms. Similarly, Figure 4A shows that the weaker contralateral occipital prestimulus power (possibly reflecting higher cortical excitability), the stronger the ERP is 
frontal electrodes $275-400 \mathrm{~ms}$. The topography and timing of this effect resembles the P3a ERP component. These observations suggest that prestimulus EEG activity modulates the activity in similar time windows and sites as conscious visibility. Ipsilateral occipital prestimulus power did not predict ERP amplitudes.
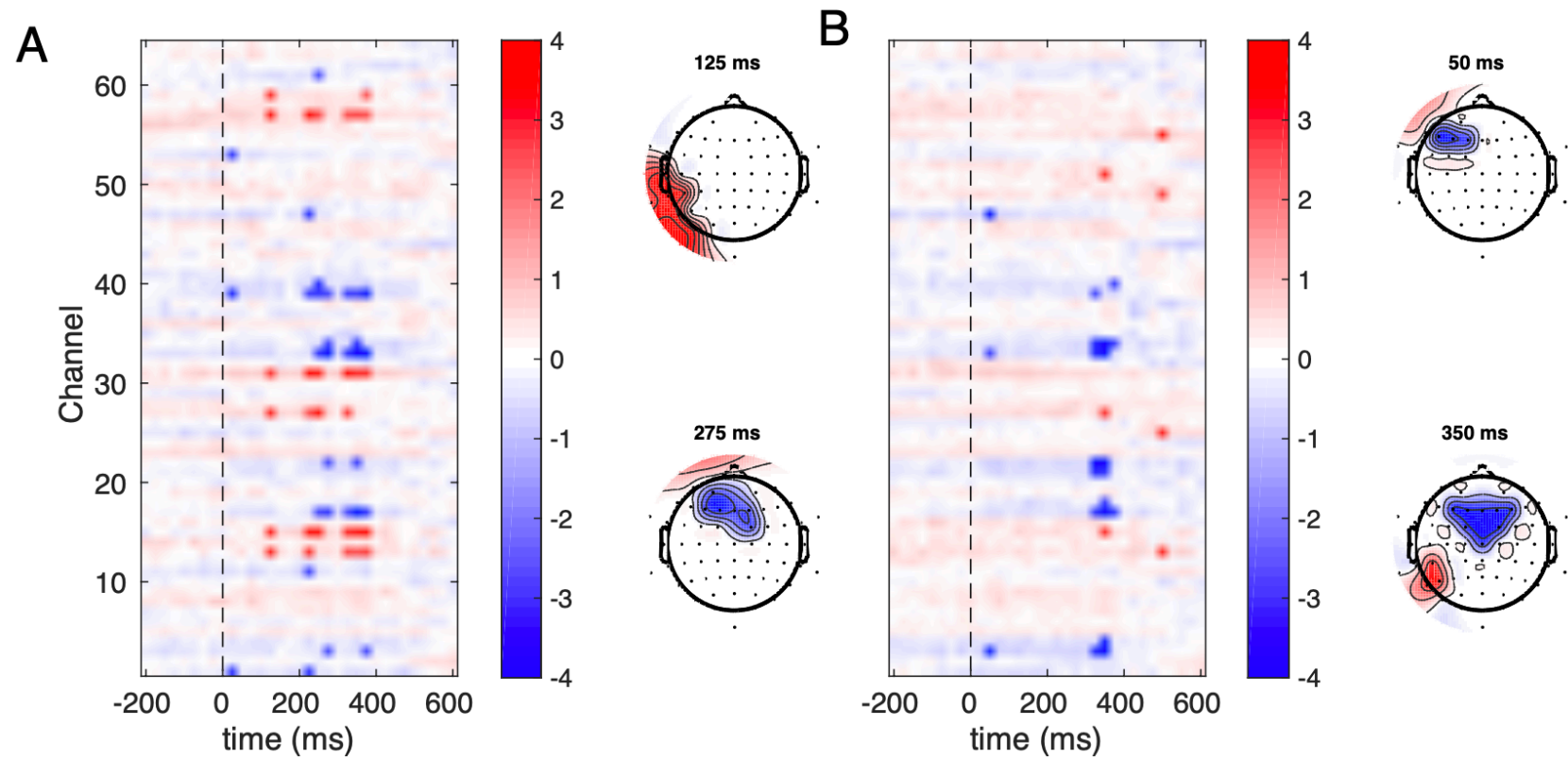

Figure 4. Influence of single-trial prestimulus oscillatory power on stimulus-evoked ERPs. A) Effect of contralateral occipital prestimulus power on ERPS when the analysis includes all trials. B) Same analysis when only subliminal trials are included. The rectangular colormap plot shows the results of the mass univariate linear mixed-effects models. Color represents the tvalue. A strong color represents statistically significant effects, as determined by TFCE. Scalp maps show the distribution of the effects at specified time points (note that the electrode locations have been transposed: electrodes over the left hemisphere are contralateral to the stimulus).

Next, we tested whether any relationship between the prestimulus oscillatory power and stimulus-evoked ERPs would be observed if the analysis was restricted to trials where the participants reported not seeing the target at all. As shown in Figure 4B, stimulus-evoked ERP amplitude at the frontal electrodes was found to be modulated by prestimulus power in the same way as previously described. Ipsilateral occipital prestimulus power did not predict ERP amplitudes when only reportedly subliminal trials were included in the analysis. In sum, these results show that on trials where the participants reported not seeing a stimulus, fluctuations in prestimulus power (in frequency/time windows that predicted subliminal perception) modulated ERP amplitudes in the frontal electrodes.

\section{Event-related spectral perturbations}

We also examined how behavioral performance was related to stimulus-evoked changes in oscillatory power (ERSPs). As shown in Figure 5B (lower panels), single-trial ERSPs were strongly modulated by stimulus visibility (very similar effects were observed for objective discrimination performance, as shown in Supplementary Figure 6). Higher visibility of the stimulus was associated with higher ERSP responses 100-400 ms after stimulus onset. Around $300 \mathrm{~ms}$ after stimulus onset, stimulus visibility correlated negatively with ERSP amplitude (roughly frequencies $7-30 \mathrm{~Hz}$ ). These observations replicate previous findings (Bareither et al., 2014; Li, Hill, \& He, 2014). 
A

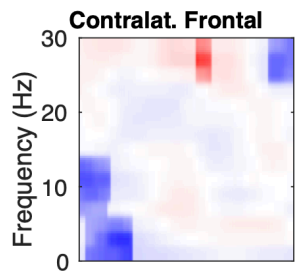

B

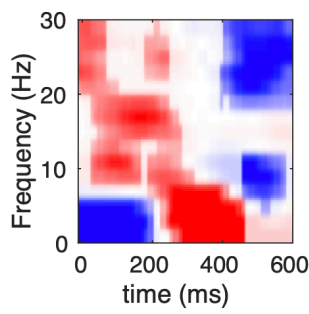

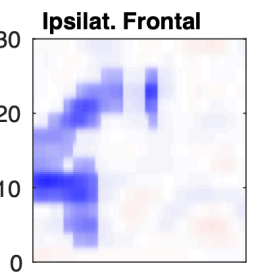
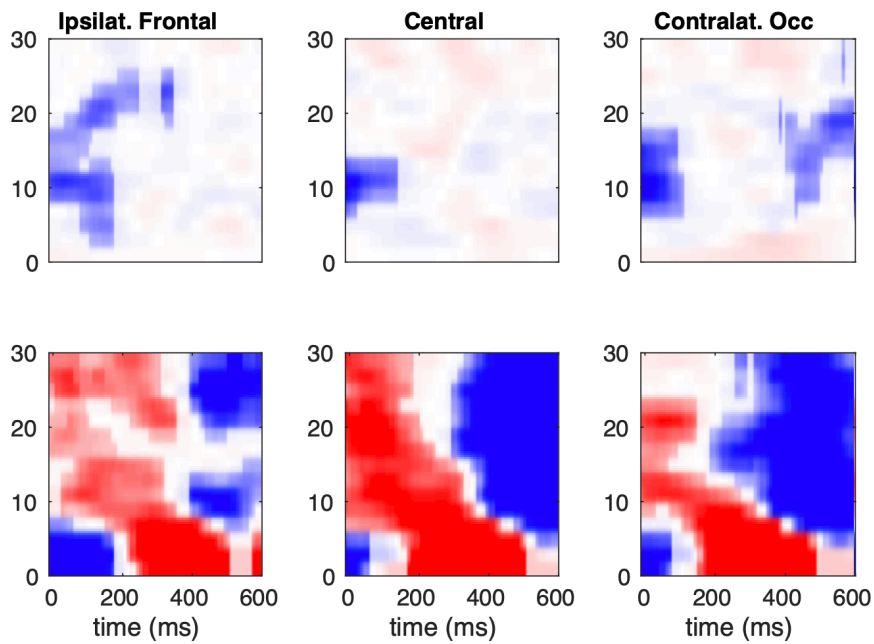

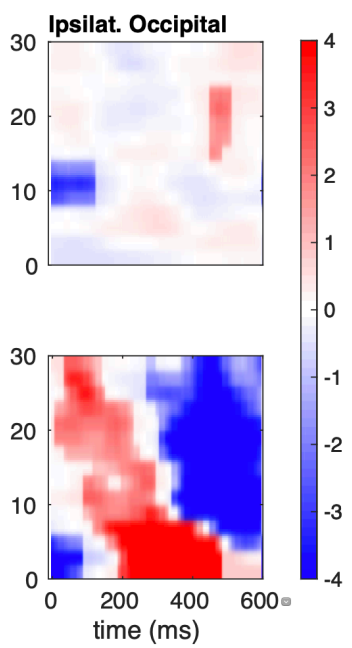

Figure 5. How ERSP amplitude predicts behavioral performance. Results of the mass univariate mixed-effects logit models where single-trial ERSP amplitudes were used to predict A) location discrimination accuracy in subliminal trials (visibility $=0$ ) and B) binarized visibility ratings (did not see $=0 ; 1-3=$ saw). Color is the $t$ value of the mixed-effects logit model. A blue color denotes a negative correlation and red a positive correlation. The faint colors are time points/frequencies where the model does not statistically significantly predict the behavioral outcome. Bright colors show statistically significant modulation according to TFCE and permutation testing. Different columns represent different clusters of electrodes.

Objective discrimination performance in single trials where the participants reported not seeing the target modulated ERSPs clearly to a lesser extent (Figure 5A). What is noteworthy is that an ERSP amplitude between 100-400 ms in occipital electrodes - where the strongest stimulusevoked activation is expected - showed very weak modulation by subliminal discrimination accuracy. In occipital electrode clusters, the strongest effect was observed after $400 \mathrm{~ms}$ of stimulus onset in the contralateral cluster (around 10-20 Hz). As seen in Figure 5A, there were also some earlier effects in the frontal areas (roughly between 0-200 ms poststimulus).

Finally, we examined how single-trial variation in prestimulus power influenced ERSPs. As earlier, we restricted this analysis to the two prestimulus correlates that predicted behavioral performance in subliminal trials. Figure 6 shows the results when the analysis included only reportedly subliminal stimuli. Figure 6 A shows how 5-9 Hz contralateral occipital prestimulus power influenced ERSPs. Remember that contralateral occipital prestimulus power was inversely related to behavioral performance in the subliminal trials (Figure 2): the lower the prestimulus power, the higher the likelihood of a correct response. As shown in Figure 6A (upper row), the pattern is different when the influence of the contralateral prestimulus power to ERSP is examined: the higher the prestimulus power, the higher the response to the stimulus is at the same frequency range $(5-9 \mathrm{~Hz})$ right after stimulus onset $(0-200 \mathrm{~ms})$. This correlation may be trivial in the sense that the result merely shows that when the power is high right before stimulus onset, it is also likely high right after stimulus onset. However, after about $300 \mathrm{~ms}$ after stimulus onset, the effect reverses into a negative correlation. Overall, the pattern of modulation by contralateral prestimulus power resembles the correlates of stimulus visibility (compare Figure 6 upper row to Figure 5 lower row). This finding is paradoxical because it seems to conflict with the relationship prestimulus power had with the behavioral discrimination accuracy: the higher the contralateral prestimulus power was, the less likely were the participants to correctly discriminate the location of a subliminal stimulus. Yet, puzzlingly, the effect of high contralateral prestimulus power on poststimulus ERSP was comparable to the correlates of subjective visibility. 
A

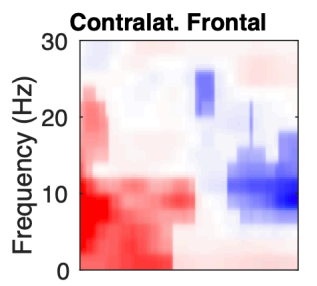

B

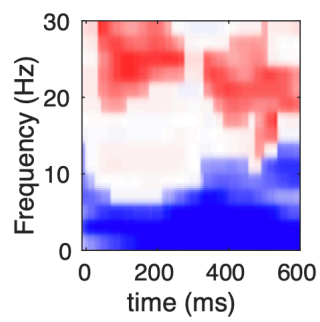

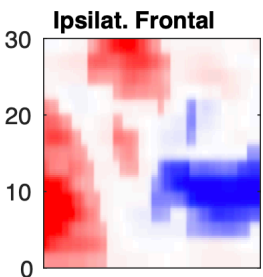

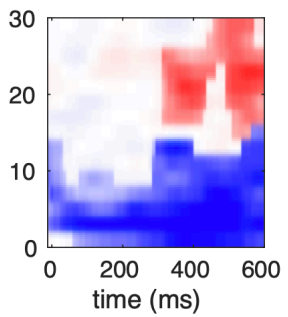

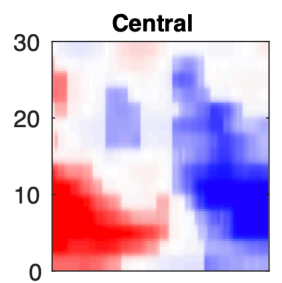
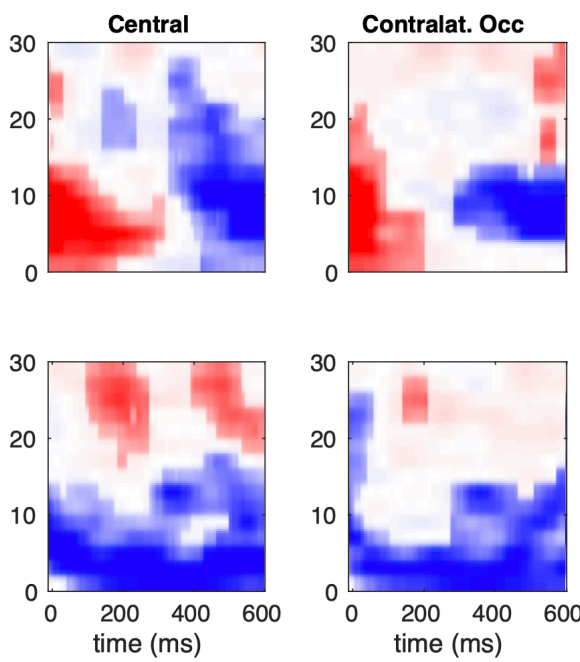

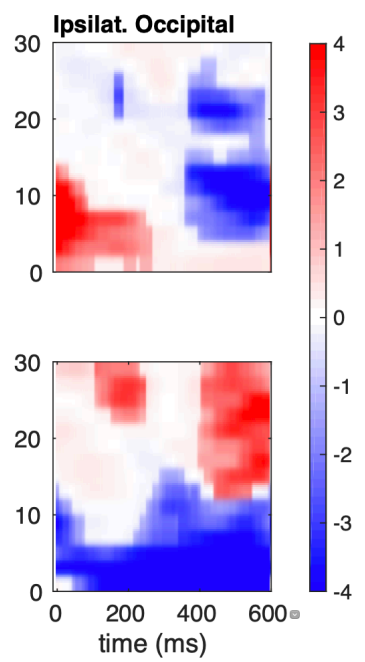

Figure 6. Influence of single-trial prestimulus oscillatory power on ERSP amplitude. A) How 5-9 $\mathrm{Hz}$ contralateral occipital prestimulus power influenced ERSPS. B) How $3 \mathrm{~Hz}$ ipsilateral occipital prestimulus power influenced ERSPS. Color is the $t$ value of the mixed-effects logit model. A blue color denotes a negative correlation and red a positive correlation. The faint colors are the time points/frequencies where the model does not statistically significantly predict the behavioral outcome. The bright colors show statistically significant modulation according to TFCE and permutation testing. Different columns represent different clusters of electrodes.

Ipsilateral occipital $3 \mathrm{~Hz}$ prestimulus activity showed a different pattern: the higher the ipsilateral occipital prestimulus power, the weaker the ERSP response in low frequencies was (Figure 6B). Weak ERSP responses at low frequencies implies that stimulus-related processing was suppressed (strong low-frequency ERSP response was associated with high stimulus visibility, Fig. $5 \mathrm{~B})$. This is consistent with the idea that accurate discrimination of stimuli has to do with suppressing unwanted activation from the ipsilateral visual field.

\section{Discussion}

We investigated what factors may explain why behavioral visual discrimination accuracy can be on average above the level of chance, even though the participants subjectively report not seeing the stimulus (i.e. "subliminal perception"). Our results reveal the following: First, subliminal perceptual performance was not completely independent of conscious introspection, as measured by the signal detection theory. Second, lateralized neural activity up to $1 \mathrm{~s}$ before stimulus presentation predicted behavioral performance even when the participants reported just making guesses. Interestingly, a similar effect was not observed for stimulus-evoked responses, although the visual stimuli participants reported not seeing evoked clear electrophysiological responses (as measured by ERP/ERSP). Third, lateralized prestimulus activity in occipital electrodes was shown to influence stimulus-evoked electrophysiological responses in a complicated way. Prestimulus activation may influence subliminal perception in part by suppressing stimulus evoked activation in the visual space where no stimulus was presented. However, by and large, the results do not support the view that trial-by-trial variation in behavioral performance in subliminal trials can be accounted for by variation in the strength of the stimulus evoked response, as previously suggested.

Our behavioral results suggest that the performance on reportedly subliminal trials was not completely independent of the participants' introspective abilities, at least based on the signal detection theoretic metrics of sensitivity and criterion (which were calculated based on subjective perceptual reports). This means that when the participants reported not seeing the stimuli, the above chance behavioral performance on these subliminal trials may nevertheless be based on signals- 
barely different from the "noise"- that the participants could introspect and base their response to. In general, signal detection theoretic framework implies that dissociations between subjective detection and forced-choice discrimination may be observed, not because there is a distinct unconscious perceptual capacity, but because the sensitivity of discrimination task is higher than the sensitivity of perceptual reports (Campion, Latto, \& Smith, 1983; King \& Dehaene, 2014; Phillips, 2018). Part of the difficulty in answering whether the signals on which the participants based their responses on subliminal trials were unconscious or not is that it requires us to artificially binarize a process that is in truth continuous. Our finding is in line with the observation by Peters and Lau (2015), who showed that when a paradigm is used which minimizes the participants' need to specify a criterion to label something as "conscious" or "unconscious", no evidence for unconscious perception is observed. Second, it could be debated whether the nature of the information on what the participants based their decision on was visual or not, but the key point is that the participants had some type of conscious access to information about the location of the stimulus on subliminal trials. Finally, objective visual discrimination behavior and subjectively experienced perception evoked nearly identical ERP/ERSP responses, suggesting that the two are not strongly dissociated.

Why did the accuracy of behavioral discrimination on subliminal trials not modulate ERP/ERSP amplitudes if prestimulus fluctuations in EEG power did influence them? Possibly the statistical power for detecting how prestimulus power influences ERPs/ERSPs is higher because the analysis was based on continuous regressors rather than on a categorical behavioral outcome (i.e., correct versus incorrect responses). Although we collected visibility ratings using a four-step scale, we decided to binarize the categories because the participants mostly used the two lower alternatives. Moreover, dichotomization of seen/not-seen ratings allowed for straightforward testing of the mechanisms of subliminal vision, which was the major goal. That is, we defined "subliminal" (or "unconscious") by equating it with the lowest visibility rating (Overgaard, 2011; Ramsøy \& Overgaard, 2004).

Previous studies have shown that, in addition to the strength of prestimulus oscillations, the pattern of prestimulus activation is a significant predictor of perceptual performance (Boncompte et al., 2016; Britz et al., 2014). Baria et al. (2017) showed that participants' behavioral responses on subliminal trials could be predicted from prestimulus activity patterns: when prestimulus activity resembled left stimulus-evoked responses, the participants were more likely to respond "left." Because we transposed our electrode locations to correspond to contralateral versus ipsilateral space, our analysis was sensitive to the spatial pattern of the evoked response. Our motivation to transpose electrode locations to contralateral versus ipsilateral space was partly that the lateralization of prestimulus power could explain how strongly the visual system responds to a visual stimulus in the contralateral visual field. This type of mechanism is unlikely to strongly explain subliminal perception in the present study because we did not observe differences in ERP/ERSP amplitudes 100-400 ms after stimulus presentation during the unconscious trials. That said, high ipsilateral prestimulus power was associated with weak low-frequency ERSP power, suggesting that accurate subliminal discrimination may in part be because of successful inhibition of noise in the ipsilateral (nontarget) hemisphere.

Early ERP waves ( $<200 \mathrm{~ms}$ after stimulus onset) are assumed to reflect the addition of sensory-evoked neural activation on top of ongoing neural activity (Mäkinen, Tiitinen, \& May, 2005; Shah et al., 2004). In addition to this mechanisms, later ERP responses are produced by asymmetric amplitude modulation of brain oscillations (Mazaheri \& Jensen, 2008; Nikulin et al., 2007). Spontaneous prestimulus neural activity may modulate ERPs through both of these mechanisms. Iemi et al. (2019) showed that while the power of prestimulus alpha- and beta-band activity influenced the earliest visual ERPs (C1 and N1 waves), the asymmetry of oscillations predominantly influenced later ERP waves. Our finding that subliminal perception modulated late ERP/ERSP components suggests that in our study the baseline-shift mechanism may have influenced subliminal perceptual performance more than variation in cortical excitability. 
Our results are at odds, at least superficially, with the interpretation that the power of prestimulus alpha merely modulates subjective detection performance and not objective discrimination (Iemi et al., 2017; Samaha et al., 2020). This discrepancy is at least partly related to the fact that we transposed the electrode locations. As shown in Supplementary Figure 3, our results do replicate the previous finding that when non-transposed electrode locations are used, subjective visibility is inversely associated with prestimulus alpha power (Iemi et al., 2017), although the effect is relatively weak. That said, we also observed that prestimulus alpha power predicted objective visual discrimination performance. However, notably, objective discrimination performance could be much better predicted when the channel locations were transposed. This suggests that part of the reason why previous studies have not have detected that prestimulus oscillatory power predicts discrimination performance is because their analysis has not examined the lateralization (or more generally the pattern) of prestimulus power.

Our results also suggest that interesting dissociations between lateralized and non-lateralized prestimulus power may exist. Lateralization of $<15 \mathrm{~Hz}$ power may contribute to visual discrimination (at least when targets are left/right lateralized, as in our study), whereas nonlateralized power may better predict reported subjective visibility. Note that although this could be interpreted as a (neural) dissociation between conscious vision and visually-guided behavior, the result does not have to imply such dissociation. Rather, non-lateralized alpha power could also only bias the reports of subjective visibility, or decision making more generally, not perception per se (Samaha et al., 2020). In the present study, when discrimination accuracy was predicted by prestimulus power of trials where participants reported consciously perceiving the stimulus (i.e. more liberal criterion), the results do not show lateralization (Supplementary Figure 7; note that this analysis is based on small number of trials). This is consistent with the claim that subjective visual perception and visually guided discrimination are not be strongly dissociated in the present data.

Based on previous studies (Koivisto \& Grassini, 2016; Lamy et al., 2009) and models of (un)conscious vision (Dehaene et al., 2006; Lamme \& Roelfsema, 2000), we expected that ERP amplitudes over visual cortical areas would correlate with both stimulus visibility and subliminal discrimination performance at around $200 \mathrm{~ms}$ poststimulus (i.e., VAN). Our results do not support this conclusion because VAN did not reach statistical significance, and it also did not correlate with subliminal discrimination. Koivisto and Grassini (2016) observed that VAN amplitude correlated with discrimination performance when the participants reported not seeing the stimulus. Unlike in the present study, Koivisto and Grassini (2016) used a four-alternative discrimination task. The higher spatial uncertainty associated with four possible stimulus positions (in contrast to two positions in the present study) enabled the use of higher contrast stimuli, which could imply a higher power to detect amplitude differences in the VAN time window. Another interesting difference between many previous studies that have detected VAN and the present study is that previous studies that have observed a strong VAN have typically used a reference electrode in the nose (e.g., Koivisto \& Grassini, 2016). Possibly, nose reference allows for better sensitivity to detect effects in posterior electrodes. Finally, unlike Koivisto and Grassini (2016), who performed their analysis on amplitudes averaged over a time window, we performed a mass univariate analysis, which weakens our statistical power for detecting small amplitude differences. In sum, we conclude that the absence of statistically significant associations between occipital ERPs and behavioral performance/prestimulus oscillations should not be taken as evidence that such effects and mechanism do not exist.

Our results also contrast previous findings that P3 amplitude is modulated by objective visual performance on subliminal trials (Salti et al., 2012, 2015). Although P3 amplitude often correlates with the conscious vision (as it did in the present study), the P3 seems to reflect the processing of task-relevant information (Pitts, Padwal, Fennelly, Martínez, \& Hillyard, 2014; Shafto \& Pitts, 2015), and decision making processes (O’Connell, Dockree, \& Kelly, 2012; Tagliabue et al., 2019), rather than sensory consciousness (Förster et al., 2020; Railo et al., 2011). 
That subliminal perception in the present study did not correlate with P3 amplitude could mean that decisions regarding subliminal stimuli were not based on the same processes as decision regarding consciously perceived stimuli. Possibly, decision on the faint subliminal percepts required longer processing time (than consciously seen stimuli), and hence modulated ERPs at later time-windows (Fig. 3 suggests that discrimination accuracy in subliminal trials modulated ERPs after $500 \mathrm{~ms}$ after stimulus onset).

Interestingly, contralateral occipital prestimulus power predicted ERP amplitude in frontal areas, when only the subliminal trials were included in the analysis. The topography and timing of this effect resembles the observed correlates of conscious vision (Fig. 3), and P3a ERP component, which has been interpreted as a correlate of orienting attention towards stimuli (Polich, 2007). This suggests that correct discrimination on subliminal trials may have to do with successfully attending the weak, conscious stimulus-related representations. Prefrontal decision-making mechanisms can be activated by subliminal stimuli (Lau \& Passingham, 2007; Van Gaal, Ridderinkhof, Scholte, \& Lamme, 2010). Studies have also reported that activity in the frontal cortical areas correlates with subliminal behavioral performance (Lau \& Passingham, 2006) and conscious vision (Persaud et al., 2011) in blindsight patients.

Our results are interesting when considered from the perspective of blindsight patients. Blindsight capacity has been traditionally explained by referring to the anatomical pathways that bypass the primary visual cortex (Ajina \& Bridge, 2018; Ajina, Pestilli, Rokem, Kennard, \& Bridge, 2015; Azzopardi \& Cowey, 1997; Cowey, 2010; Danckert \& Rossetti, 2005; Weiskrantz, Warrington, Sanders, \& Marshall, 1974). However, whether blindsight patients' behavioral responding is based on completely unconscious percepts (Campion et al., 1983; Phillips, 2018), and whether similar mechanisms guide behavior in neurologically healthy individuals (Allen et al., 2020; Hurme, Koivisto, Henriksson, \& Railo, 2020; Hurme, Koivisto, Revonsuo, \& Railo, 2017, 2019; Lloyd, Abrahamyan, \& Harris, 2013) remains debated. An interesting open question is to what extent prestimulus processes also contribute to blindsight. Previous studies on blindsight patients have focused on stimulus-evoked neural activation but have not considered what neural mechanisms modulate trial-by-trial variability in blindsight capacity. The present results resonate nicely with Ko and Lau (2012), who suggested that blindsight patients fail to adapt a proper criterion of conscious perception after a lesion.

In conclusion, our results show that subliminal perception should not be seen as a passive, automatic perceptual capacity whose neural mechanism relates to how strongly the visual system responds to a stimulus. Instead, accurate objective visual discrimination in subliminal trials depends on the state of the brain right before the stimulus is presented. Our results also emphasize that conscious vision seems to be tightly linked with behavior, as performance on reportedly unconscious trials may actually depend on processes that a participant can consciously introspect.

\section{References}

Ajina, S., \& Bridge, H. (2018). Blindsight relies on a functional connection between hMT + and the lateral geniculate nucleus, not the pulvinar. PLoS Biology. https://doi.org/10.1371/journal.pbio.2005769

Ajina, S., Pestilli, F., Rokem, A., Kennard, C., \& Bridge, H. (2015). Human blindsight is mediated by an intact geniculo-extrastriate pathway. ELife. https://doi.org/10.7554/eLife.08935

Allen, C., Viola, T., Irvine, E., Sedgmond, J., Castle, H., Gray, R., \& Chambers, C. D. (2020). Causal manipulation of feed-forward and recurrent processing differentially affects measures of consciousness. Neuroscience of Consciousness. https://doi.org/10.1093/nc/niaa015

Azzopardi, P., \& Cowey, A. (1997). Is blindsight like normal, near-threshold vision? Proceedings of the National Academy of Sciences of the United States of America. https://doi.org/10.1073/pnas.94.25.14190

Bareither, I., Chaumon, M., Bernasconi, F., Villringer, A., \& Busch, N. A. (2014). Invisible visual 
stimuli elicit increases in alpha-band power. Journal of Neurophysiology. https://doi.org/10.1152/jn.00550.2013

Baria, A. T., Maniscalco, B., \& He, B. J. (2017). Initial-state-dependent, robust, transient neural dynamics encode conscious visual perception. PLoS Computational Biology. https://doi.org/10.1371/journal.pcbi.1005806

Benwell, C. S. Y., Tagliabue, C. F., Veniero, D., Cecere, R., Savazzi, S., \& Thut, G. (2017). Prestimulus EEG power predicts conscious awareness but not objective visual performance. ENeuro. https://doi.org/10.1523/ENEURO.0182-17.2017

Boncompte, G., Villena-González, M., Cosmelli, D., \& López, V. (2016). Spontaneous alpha power lateralization predicts detection performance in an un-cued signal detection task. PLoS ONE. https://doi.org/10.1371/journal.pone.0160347

Brainard, D. H. (1997). The Psychophysics Toolbox. Spatial Vision. https://doi.org/10.1163/156856897X00357

Britz, J., Hernàndez, L. D., Ro, T., \& Michel, C. M. (2014). EEG-microstate dependent emergence of perceptual awareness. Frontiers in Behavioral Neuroscience. https://doi.org/10.3389/fnbeh.2014.00163

Campion, J., Latto, R., \& Smith, Y. M. (1983). Is blindsight an effect of scattered light, spared cortex, and near-threshold vision? Behavioral and Brain Sciences. https://doi.org/10.1017/S0140525X00016861

Cowey, A. (2010). The blindsight saga. Experimental Brain Research. https://doi.org/10.1007/s00221-009-1914-2

Danckert, J., \& Rossetti, Y. (2005). Blindsight in action: What can the different sub-types of blindsight tell us about the control of visually guided actions? Neuroscience and Biobehavioral Reviews. https://doi.org/10.1016/j.neubiorev.2005.02.001

Dehaene, S., \& Changeux, J. P. (2011). Experimental and Theoretical Approaches to Conscious Processing. Neuron. https://doi.org/10.1016/j.neuron.2011.03.018

Dehaene, S., Changeux, J. P., Naccache, L., Sackur, J., \& Sergent, C. (2006). Conscious, preconscious, and subliminal processing: a testable taxonomy. Trends in Cognitive Sciences. https://doi.org/10.1016/j.tics.2006.03.007

Förster, J., Koivisto, M., \& Revonsuo, A. (2020). ERP and MEG correlates of visual consciousness: The second decade. Consciousness and Cognition. https://doi.org/10.1016/j.concog.2020.102917

Gaillard, R., Dehaene, S., Adam, C., Clémenceau, S., Hasboun, D., Baulac, M., ... Naccache, L. (2009). Converging intracranial markers of conscious access. PLoS Biology. https://doi.org/10.1371/journal.pbio.1000061

Grandchamp, R., \& Delorme, A. (2011). Single-trial normalization for event-related spectral decomposition reduces sensitivity to noisy trials. Frontiers in Psychology. https://doi.org/10.3389/fpsyg.2011.00236

Hannula, D. E., Simons, D. J., \& Cohen, N. J. (2005). Imaging implicit perception: promise and pitfalls. Nature Reviews Neuroscience. https://doi.org/10.1038/nrn1630

Hurme, M., Koivisto, M., Henriksson, L., \& Railo, H. (2020). Neuronavigated TMS of early visual cortex eliminates unconscious processing of chromatic stimuli. Neuropsychologia. https://doi.org/10.1016/j.neuropsychologia.2019.107266

Hurme, M., Koivisto, M., Revonsuo, A., \& Railo, H. (2017). Early processing in primary visual cortex is necessary for conscious and unconscious vision while late processing is necessary only for conscious vision in neurologically healthy humans. NeuroImage. https://doi.org/10.1016/j.neuroimage.2017.02.060

Hurme, M., Koivisto, M., Revonsuo, A., \& Railo, H. (2019). V1 activity during feedforward and early feedback processing is necessary for both conscious and unconscious motion perception. NeuroImage. https://doi.org/10.1016/j.neuroimage.2018.10.058 
Iemi, L., \& Busch, N. A. (2018). Moment-to-moment fluctuations in neuronal excitability bias subjective perception rather than strategic decision-making. ENeuro. https://doi.org/10.1523/ENEURO.0430-17.2018

Iemi, L., Busch, N. A., Laudini, A., Haegens, S., Samaha, J., Villringer, A., \& Nikulin, V. V. (2019). Multiple mechanisms link prestimulus neural oscillations to sensory responses. ELife. https://doi.org/10.7554/eLife.43620

Iemi, L., Chaumon, M., Crouzet, S. M., \& Busch, N. A. (2017). Spontaneous neural oscillations bias perception by modulating baseline excitability. Journal of Neuroscience. https://doi.org/10.1523/JNEUROSCI.1432-16.2016

Jensen, O., Bonnefond, M., \& VanRullen, R. (2012). An oscillatory mechanism for prioritizing salient unattended stimuli. Trends in Cognitive Sciences. https://doi.org/10.1016/j.tics.2012.03.002

King, J. R., \& Dehaene, S. (2014). A model of subjective report and objective discrimination as categorical decisions in a vast representational space. Philosophical Transactions of the Royal Society B: Biological Sciences. https://doi.org/10.1098/rstb.2013.0204

Kloosterman, N. A., De Gee, J. W., Bergner, M. W., Lindenberger, U., Garrett, D. D., \& Fahrenfort, J. J. (2019). Humans strategically shift decision bias by flexibly adjusting sensory evidence accumulation. ELife. https://doi.org/10.7554/eLife.37321

Ko, Y., \& Lau, H. (2012). A detection theoretic explanation of blindsight suggests a link between conscious perception and metacognition. Philosophical Transactions of the Royal Society B: Biological Sciences. https://doi.org/10.1098/rstb.2011.0380

Koivisto, M., \& Grassini, S. (2016). Neural processing around $200 \mathrm{~ms}$ after stimulus-onset correlates with subjective visual awareness. Neuropsychologia. https://doi.org/10.1016/j.neuropsychologia.2016.02.024

Kouider, S., \& Dehaene, S. (2007). Levels of processing during non-conscious perception: A critical review of visual masking. In Philosophical Transactions of the Royal Society B: Biological Sciences. https://doi.org/10.1098/rstb.2007.2093

Kouider, S., Dehaene, S., Jobert, A., \& Le Bihan, D. (2007). Cerebral bases of subliminal and supraliminal priming during reading. Cerebral Cortex. https://doi.org/10.1093/cercor/bhl110

Lamme, V. A. F., \& Roelfsema, P. R. (2000). The distinct modes of vision offered by feedforward and recurrent processing. Trends in Neurosciences. https://doi.org/10.1016/S01662236(00)01657-X

Lamy, D., Salti, M., \& Bar-Haim, Y. (2009). Neural correlates of subjective awareness and unconscious processing: An ERP study. Journal of Cognitive Neuroscience. https://doi.org/10.1162/jocn.2009.21064

Lau, H. C., \& Passingham, R. E. (2006). Relative blindsight in normal observers and the neural correlate of visual consciousness. Proceedings of the National Academy of Sciences of the United States of America. https://doi.org/10.1073/pnas.0607716103

Lau, H. C., \& Passingham, R. E. (2007). Unconscious activation of the cognitive control system in the human prefrontal cortex. Journal of Neuroscience. https://doi.org/10.1523/JNEUROSCI.4335-06.2007

Li, Q., Hill, Z., \& He, B. J. (2014). Spatiotemporal dissociation of brain activity underlying subjective awareness, objective performance and confidence. Journal of Neuroscience. https://doi.org/10.1523/JNEUROSCI.1820-13.2014

Limbach, K., \& Corballis, P. M. (2016). Prestimulus alpha power influences response criterion in a detection task. Psychophysiology. https://doi.org/10.1111/psyp.12666

Lloyd, D. A., Abrahamyan, A., \& Harris, J. A. (2013). Brain-stimulation induced blindsight: Unconscious vision or response bias? PLoS ONE. https://doi.org/10.1371/journal.pone.0082828

Mäkinen, V., Tiitinen, H., \& May, P. (2005). Auditory event-related responses are generated 
independently of ongoing brain activity. NeuroImage.

https://doi.org/10.1016/j.neuroimage.2004.10.020

Maris, E., \& Oostenveld, R. (2007). Nonparametric statistical testing of EEG- and MEG-data. Journal of Neuroscience Methods. https://doi.org/10.1016/j.jneumeth.2007.03.024

Mathewson, K. E., Gratton, G., Fabiani, M., Beck, D. M., \& Ro, T. (2009). To see or not to see: Prestimulus $\alpha$ phase predicts visual awareness. Journal of Neuroscience. https://doi.org/10.1523/JNEUROSCI.3963-08.2009

Mazaheri, A., \& Jensen, O. (2008). Asymmetric amplitude modulations of brain oscillations generate slow evoked responses. Journal of Neuroscience. https://doi.org/10.1523/JNEUROSCI.1631-08.2008

Muckli, L., \& Petro, L. S. (2013). Network interactions: Non-geniculate input to V1. Current Opinion in Neurobiology. https://doi.org/10.1016/j.conb.2013.01.020

Nikulin, V. V., Linkenkaer-Hansen, K., Nolte, G., Lemm, S., Müller, K. R., Ilmoniemi, R. J., \& Curio, G. (2007). A novel mechanism for evoked responses in the human brain. European Journal of Neuroscience. https://doi.org/10.1111/j.1460-9568.2007.05553.x

O’Connell, R. G., Dockree, P. M., \& Kelly, S. P. (2012). A supramodal accumulation-to-bound signal that determines perceptual decisions in humans. Nature Neuroscience. https://doi.org/10.1038/nn.3248

Overgaard, M. (2011). Visual experience and blindsight: A methodological review. Experimental Brain Research. https://doi.org/10.1007/s00221-011-2578-2

Pernet, C. R., Chauveau, N., Gaspar, C., \& Rousselet, G. A. (2011). LIMO EEG: A toolbox for hierarchical linear modeling of electroencephalographic data. Computational Intelligence and Neuroscience. https://doi.org/10.1155/2011/831409

Persaud, N., Davidson, M., Maniscalco, B., Mobbs, D., Passingham, R. E., Cowey, A., \& Lau, H. (2011). Awareness-related activity in prefrontal and parietal cortices in blindsight reflects more than superior visual performance. NeuroImage. https://doi.org/10.1016/j.neuroimage.2011.06.081

Peters, M. A. K., \& Lau, H. (2015). Human observers have optimal introspective access to perceptual processes even for visually masked stimuli. ELife. https://doi.org/10.7554/eLife.09651

Phillips, I. (2018). Unconscious Perception Reconsidered. Analytic Philosophy. https://doi.org/10.1111/phib.12135

Pitts, M. A., Padwal, J., Fennelly, D., Martínez, A., \& Hillyard, S. A. (2014). Gamma band activity and the $\mathrm{P} 3$ reflect post-perceptual processes, not visual awareness. NeuroImage. https://doi.org/10.1016/j.neuroimage.2014.07.024

Polich, J. (2007). Updating P300: An integrative theory of P3a and P3b. Clinical Neurophysiology. https://doi.org/10.1016/j.clinph.2007.04.019

Railo, H., Koivisto, M., \& Revonsuo, A. (2011). Tracking the processes behind conscious perception: A review of event-related potential correlates of visual consciousness. Consciousness and Cognition. https://doi.org/10.1016/j.concog.2011.03.019

Ramsøy, T. Z., \& Overgaard, M. (2004). Introspection and subliminal perception. Phenomenology and the Cognitive Sciences. https://doi.org/10.1023/b:phen.0000041900.30172.e8

Salti, M., Bar-Haim, Y., \& Lamy, D. (2012). The P3 component of the ERP reflects conscious perception, not confidence. Consciousness and Cognition. https://doi.org/10.1016/j.concog.2012.01.012

Salti, M., Monto, S., Charles, L., King, J. R., Parkkonen, L., \& Dehaene, S. (2015). Distinct cortical codes and temporal dynamics for conscious and unconscious percepts. ELife. https://doi.org/10.7554/eLife.05652

Samaha, J., Iemi, L., Haegens, S., \& Busch, N. A. (2020). Spontaneous Brain Oscillations and Perceptual Decision-Making. Trends in Cognitive Sciences. 
https://doi.org/10.1016/j.tics.2020.05.004

Samaha, J., Iemi, L., \& Postle, B. R. (2017). Prestimulus alpha-band power biases visual discrimination confidence, but not accuracy. Consciousness and Cognition. https://doi.org/10.1016/j.concog.2017.02.005

Sandberg, K., Timmermans, B., Overgaard, M., \& Cleeremans, A. (2010). Measuring consciousness: Is one measure better than the other? Consciousness and Cognition. https://doi.org/10.1016/j.concog.2009.12.013

Schroeder, C. E., \& Lakatos, P. (2009). Low-frequency neuronal oscillations as instruments of sensory selection. Trends in Neurosciences. https://doi.org/10.1016/j.tins.2008.09.012

Shafto, J. P., \& Pitts, M. A. (2015). Neural signatures of conscious face perception in an inattentional blindness paradigm. Journal of Neuroscience. https://doi.org/10.1523/JNEUROSCI.0145-15.2015

Shah, A. S., Bressler, S. L., Knuth, K. H., Ding, M., Mehta, A. D., Ulbert, I., \& Schroeder, C. E. (2004). Neural Dynamics and the Fundamental Mechanisms of Event-related Brain Potentials. Cerebral Cortex. https://doi.org/10.1093/cercor/bhh009

Smith, S. M., \& Nichols, T. E. (2009). Threshold-free cluster enhancement: Addressing problems of smoothing, threshold dependence and localisation in cluster inference. NeuroImage. https://doi.org/10.1016/j.neuroimage.2008.03.061

Supèr, H., Spekreijse, H., \& Lamme, V. A. F. (2001). Two distinct modes of sensory processing observed in monkey primary visual cortex (VI). Nature Neuroscience. https://doi.org/10.1038/85170

Tagliabue, C. F., Veniero, D., Benwell, C. S. Y., Cecere, R., Savazzi, S., \& Thut, G. (2019). The EEG signature of sensory evidence accumulation during decision formation closely tracks subjective perceptual experience. Scientific Reports. https://doi.org/10.1038/s41598-01941024-4

Thut, G., Nietzel, A., Brandt, S. A., \& Pascual-Leone, A. (2006). $\alpha$-Band electroencephalographic activity over occipital cortex indexes visuospatial attention bias and predicts visual target detection. Journal of Neuroscience. https://doi.org/10.1523/JNEUROSCI.0875-06.2006

Van Gaal, S., Ridderinkhof, K. R., Scholte, H. S., \& Lamme, V. A. F. (2010). Unconscious activation of the prefrontal no-go network. Journal of Neuroscience. https://doi.org/10.1523/JNEUROSCI.2992-09.2010

Vanrullen, R. (2007). The power of the feed-forward sweep. Advances in Cognitive Psychology. https://doi.org/10.2478/v10053-008-0022-3

VanRullen, R., \& Koch, C. (2003). Visual selective behavior can be triggered by a feed-forward process. Journal of Cognitive Neuroscience. https://doi.org/10.1162/089892903321208141

Watson, A. B., \& Pelli, D. G. (1983). Quest: A Bayesian adaptive psychometric method. Perception \& Psychophysics. https://doi.org/10.3758/BF03202828

Weiskrantz, L., Warrington, E. K., Sanders, M. D., \& Marshall, J. (1974). Visual capacity in the hemianopic field following a restricted occipital ablation. Brain, 97(4), 709-728. https://doi.org/10.1093/brain/97.4.709

Worden, M. S., Foxe, J. J., Wang, N., \& Simpson, G. V. (2000). Anticipatory biasing of visuospatial attention indexed by retinotopically specific alpha-band electroencephalography increases over occipital cortex. The Journal of Neuroscience: The Official Journal of the Society for Neuroscience. https://doi.org/10.1523/jneurosci.20-06-j0002.2000 


\section{Supplementary information}
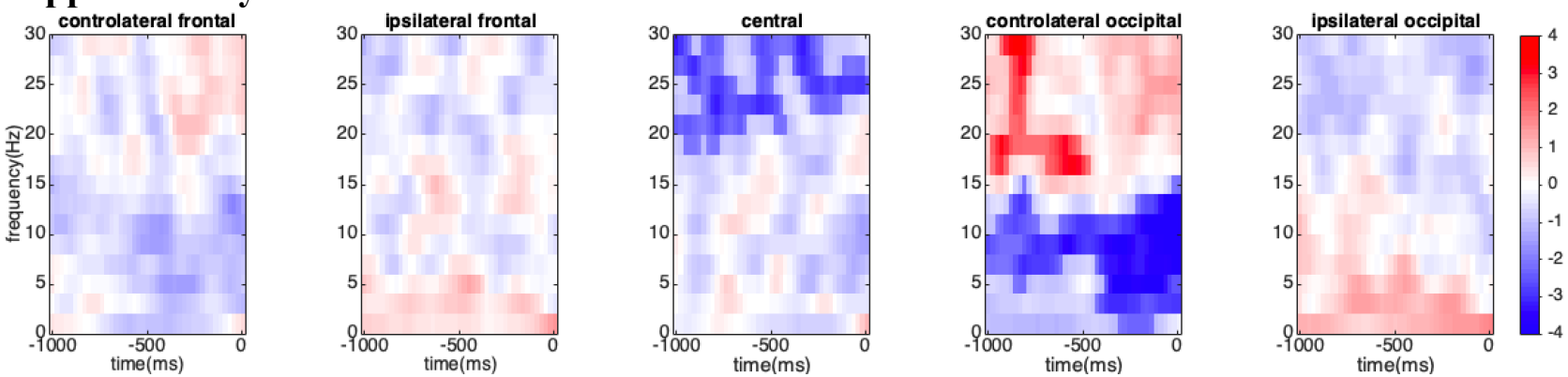

Supplementary Figure 1. Predicting the accuracy of objective discrimination based on prestimulus power. This analysis includes all trials (i.e., conscious and subliminal). Color represents the $t$ value. A bright color represents statistically significant effects according to cluster mass permutations test (not TFCE, as in main analyses).
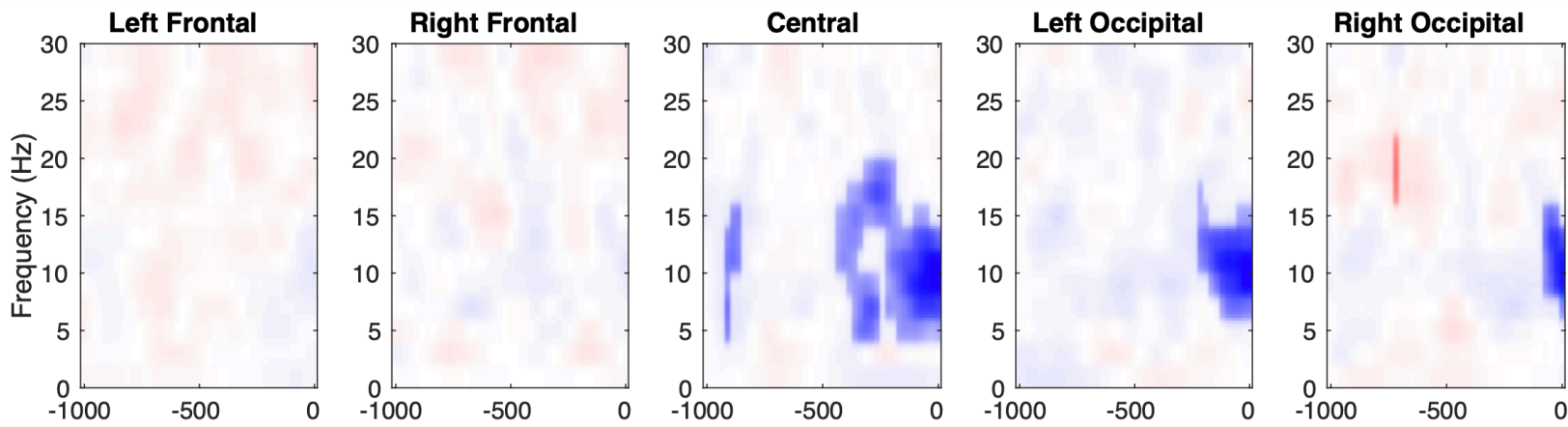

Supplementary Figure 2. Nontransposed electrode locations: Predicting discrimination accuracy on subliminal trials using prestimulus power (only trials with visibility rating $=0$ included). A bright color represents statistically significant effects (multiple-comparison correction with 1000 permutations, and TFCE).
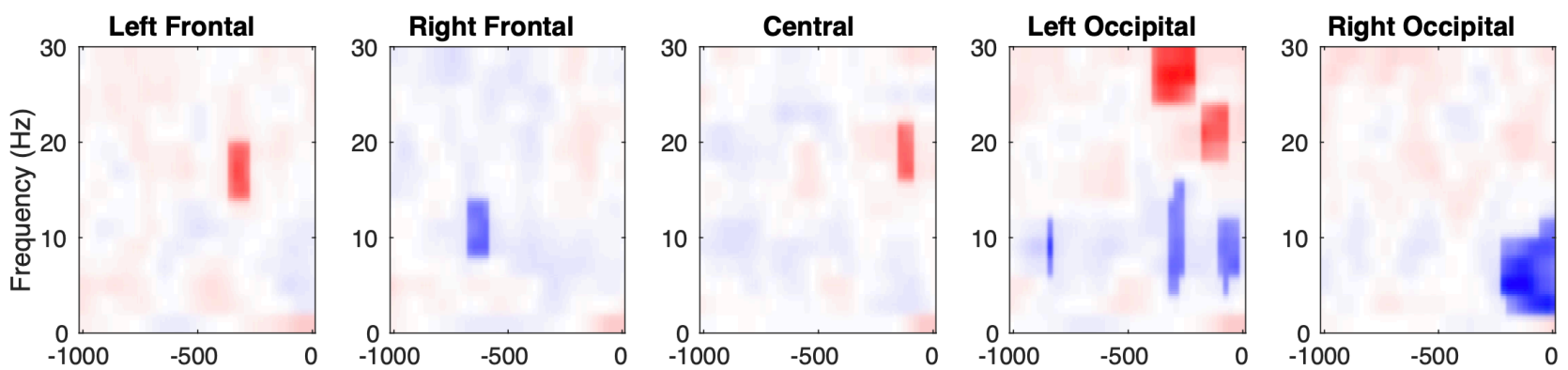

Supplementary Figure 3. Nontransposed electrode locations: Predicting binarized visibility rating using prestimulus power. A bright color represents statistically significant effects (multiplecomparison correction with 1000 permutations, and TFCE). 

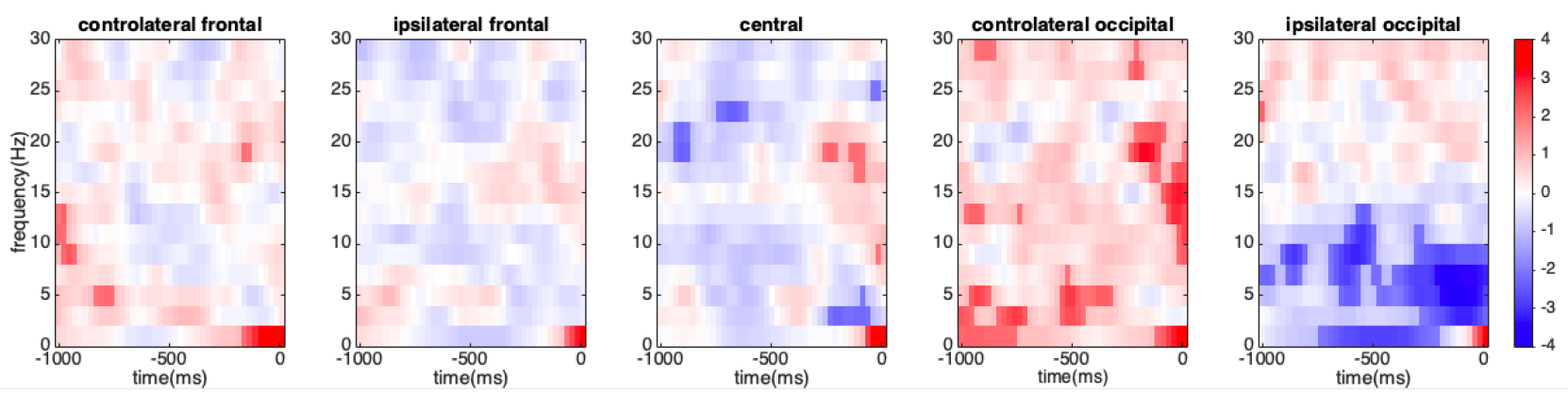

Supplementary Figure 4. Predicting visibility (subliminal vs. conscious) based on prestimulus power when only the trials where correct forced-choice answer was given are included. A bright color represents statistically significant effects $(\mathrm{p}<.05$; not corrected for multiple comparisons/no TFCE).
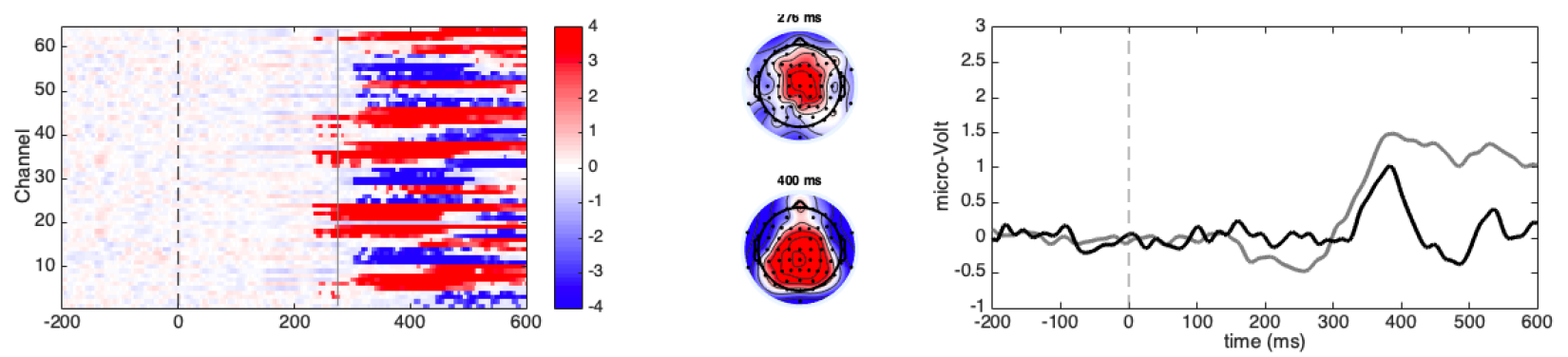

Supplementary Figure 5. Objective discrimination performance, when both consciously seen and not seen trials are included in the analysis, modulates ERPs very similarly as subjective visibility. Grey vertical line is $275 \mathrm{~ms}$ time point.
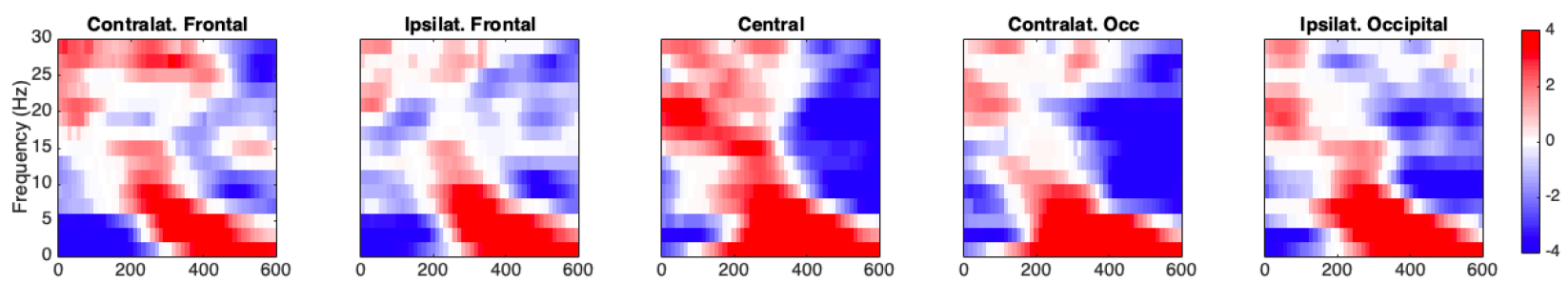

Supplementary Figure 6. Objective discrimination performance, when both consciously seen and not seen trials are included in the analysis, modulates ERSPs very similarly as subjective visibility.
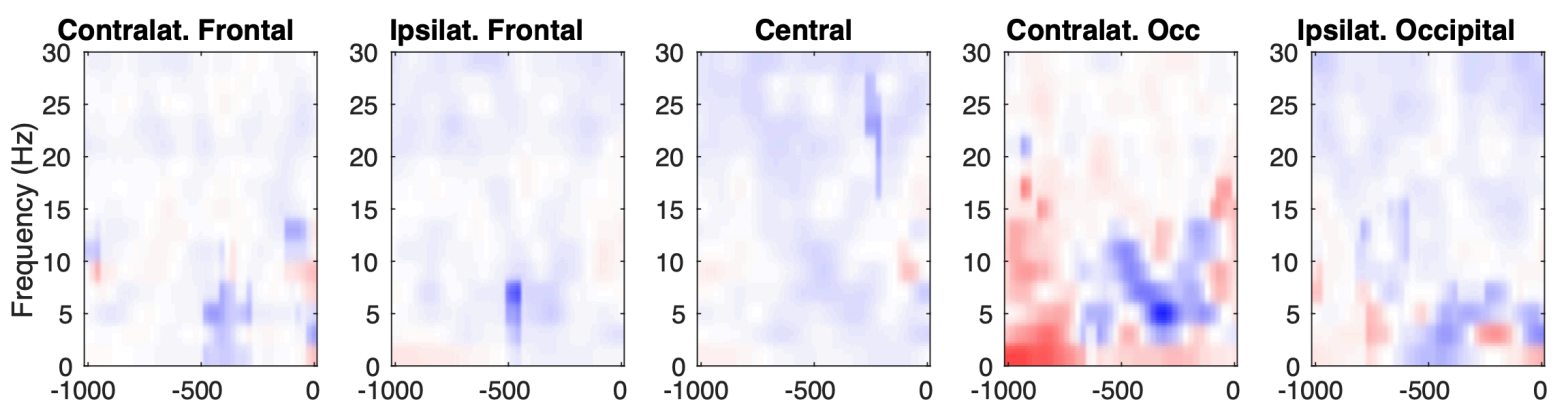

Supplementeray Figure 7. Predicting discrimination accuracy on consciously seen trials using prestimulus power. A bright color represents statistically significant effects (multiple-comparison correction with 1000 permutations, and TFCE). 